\title{
Modelling of Dynamic Problems in Biomechanics
}

\author{
I. Petrov ${ }^{1}$, Y. Bolotskikh ${ }^{1}$ and A. Vasyukov ${ }^{1}$ \\ ${ }^{1}$ Department of Computer Science, Moscow Institute of Physics and Technology, \\ Dolgoprudny 141700, Russia
}

\begin{abstract}
This paper is devoted to solving of dynamic problems in biomechanics that require detailed study of fast processes. Numerical method of characteristics is used to model the temporal development of the processes with high accuracy.
\end{abstract}

Key words: method of characteristics, biomechanics, cataract extraction, brain injury, heart contraction

AMS subject classification: 65M25, 92C10

\section{Introduction}

Mathematical modelling is currently one of the most important medical research areas whether it concerns normal physiological or pathological processes. Mathematical modelling as a research method has a number of clear advantages: availability of numerical experiment, possibility to use a wide range of main parameters and resulting full impression of processes occurring throughout the system.

Using numerical methods in order to solve the biomechanical problems allows to study traumatologic and surgical processes in different organs and to understand the underlying patterns and mechanisms. Therefore, it makes possible to determine key trauma-causing factors and features that influence the development of a disease. The numerical modelling results can be used by clinicians to enhance practical guidelines quality and to develop new diagnostics and therapy methods as well as create protective means against trauma.

\footnotetext{
${ }^{*}$ Corresponding author. E-mail: vasyukov@gmail.com
} 
In order to successfully solve practical problems the research should be carried out in collaboration with clinicians. In particular, this research concentrates on few biomechanical problems. The craniocerebral injury problem was set up together with Sklifosovsky Scientific Research Institute of Emergency Medicine and Burdenko Principal Military Clinical Hospital. The laser cataract extraction problem was posed jointly with Fyodorov Eye Microsurgery Institution.

\subsection{Methods of characteristics for biomechanics fast processes}

This paper is devoted to dynamic biomechanical problems concerning traumatologic and surgical processes. These problems specific feature is that the occurring processes often have a wave nature with very small characteristic times.

Unfortunately, most of the papers in this research area which are known to the authors pay insufficient attention to the processes temporal development. It is the final state of the system that comes under close examination in the mathematical modelling. The tools used usually include a variety of well accepted finite elements methods which provide high accuracy for static problems (see, for example $[1,2,3]$ ).

This approach can be improved. Fast mechanical processes in biological tissues such as shock wave propagation and interaction are commonly described by systems of hyperbolic equations as shown below.

Grid-characteristic numerical methods and difference schemes of the types formulated by Godunov, Kogan, Fedorenko can be used to study the development of fast processes with small characteristic times. These methods and schemes were initially designed for solving systems of hyperbolic equations and take into account equations specific features to achieve high accuracy for the solution of wave nature problems including the wavefront interactions.

A successful application of grid-characteristic numerical methods to biomechanical problems can be demonstrated on the example of craniocerebral injury modelling (as described below). Accurate consideration of shock wave interactions allows predicting of the brain maximum damage areas which are caused by shear waves interactions resulted in vessel ruptures. This process has an intrinsically dynamic nature, and its examination by any other means is highly hampered.

\section{Mathematical models and methods used}

\subsection{Mechanical models}

Mechanical properties of biological medium under relatively small strain which is considered as a continuous medium under the action of shock loads can be described using common equations for deformable rigid body. This approach should be used carefully, because biological tissues modelling often requires taking into account fluids, cavities and rigid bodies with very specific rheology. However, for many problems it is reasonable and allows to achieve good results. 
For this research we used the equations system of the linear elasticity theory [4]:

$$
\begin{gathered}
\rho \cdot \dot{v}_{i}=\nabla_{j} \cdot \sigma_{i j}, \\
\sigma_{i j}=q_{i j k l} \cdot e_{k l}+F_{i j} .
\end{gathered}
$$

Here (2.1) are the equations on motion and (2.2) are the rheological relations. In these equations $\rho$ is the medium density, $v_{i}$ are the displacement velocity components, $\sigma_{i j}$ and $e_{i j}$ are the components of the stress tensors and the strain velocities, $\nabla_{j}$ is the covariance derivative with respect to the $j$ th coordinate and $F_{i j}$ is the right-hand side.

The tensor $q_{i j k l}$ determines the rheology of the medium. In case of a linearly elastic body its components are expressed in terms of two independent Lame constants $\lambda$ and $\mu$ :

$$
q_{i j k l}=\lambda \cdot \delta_{i j} \cdot \delta_{k l}+\mu\left(\delta_{i k} \cdot \delta_{j l}+\delta_{i l} \cdot \delta_{j k}\right) .
$$

We also used Maxwell's viscoelastic body model.

The density is determined from the equation of state $\rho=\rho_{0} e^{(p / K)}$, where $p=-\frac{1}{3} \sum \sigma_{k k}$ is a pressure and $K=\lambda+\frac{2}{3} \mu$ is a uniform compression coefficient.

These equations are suitable for modelling wave processes in continuous medium under relatively small shock loads. Wave processes have small characteristic times, so deformations are small during the numerical experiment. These assumptions allow us to use the model of linear elastic body.

Equations of motion and rheological relations stated above can be written in the matrix form:

$$
\frac{\partial}{\partial t} u+A_{1} \frac{\partial}{\partial x_{1}} u+A_{2} \frac{\partial}{\partial x_{2}} u+A_{3} \frac{\partial}{\partial x_{3}} u=f .
$$

Here $u=\left(v_{1}, v_{2}, v_{3}, \sigma_{11}, \sigma_{12}, \sigma_{13}, \sigma_{22}, \sigma_{23}, \sigma_{33}\right)^{T}$ is the vector of variables, $f$ is the right-hand side of the same dimension, $A_{i}$ are the matrices of ninth order (their explicit form can be found in [7]), $x_{i}$ are independent three-dimensional variables and $t$ is the time.

If the matrices $A_{i}$ have nine real eigenvalues, then the system is called hyperbolic and its solutions correspond to the processes that are usually called wave processes. That is, this equation describes the propagation of perturbations along the characteristic cones in the space $\left(x_{1}, x_{2}, x_{3}, t\right)$.

\subsection{Grid-characteristic numerical methods}

Grid-characteristic numerical methods were used for solving the system of hyperbolic equations (2.4).

One of the widely used approaches to solving three-dimensional system (2.4) is splitting it by space variables. In this case it can be replaced with four one-dimensional systems:

$$
\begin{aligned}
& \frac{\partial}{\partial t} u+A_{1} \frac{\partial}{\partial x_{1}} u=0, \\
& \frac{\partial}{\partial t} u+A_{2} \frac{\partial}{\partial x_{2}} u=0,
\end{aligned}
$$




$$
\begin{gathered}
\frac{\partial}{\partial t} u+A_{3} \frac{\partial}{\partial x_{3}} u=0, \\
\frac{\partial}{\partial t} u=f .
\end{gathered}
$$

These systems should be solved in their course, where a solution of the previous system should be used as the initial state to solve the next one. This approach allows to simplify numerical implementation and obtain better computation performance.

Each one-dimensional system is solved using common grid-characteristic numerical methods. For a system of equations with a single space variable

$$
\frac{\partial}{\partial t} u+A \frac{\partial}{\partial x} u=f
$$

the solution is sought in the form of a grid function $u_{m}^{n}$ defined at the points of the computational grid $x_{m}=m h, t^{n}=n \tau$, where $h$ and $\tau$ are the grid size with respect to space and time.

Monotonous first order scheme (Courant-Isaacson-Rees). This scheme is constructed on the basis of the analysis of system (2.9) characteristics behavior and yields the following formulas (see [5]):

$$
u_{m}^{n+1}=u_{m}^{n}-\frac{\tau}{h} \Omega^{-1} \Lambda^{+} \Omega\left(u_{m+1}^{n}-u_{m}^{n}\right)-\frac{\tau}{h} \Omega^{-1} \Lambda^{-} \Omega\left(u_{m}^{n} u_{m-1}^{n}\right) .
$$

Here $\Omega$ is the matrix of left-hand eigenvectors of matrix $A, \Lambda^{ \pm}$is the diagonal matrix of corresponding eigenvalues.

Scheme (2.10) has the order of approximation $O(h, \tau)$, it is monotone and has the minimal approximation viscosity among the first-order monotone schemes, which is very important for the calculation of dynamical processes in inhomogeneous media.

Second order scheme (Lax-Wendroff). This is the only central scheme of the second order of approximation on a three-point stencil (i.e., a standard scheme using the values at the points $m-1, m, m+1)$ :

$$
u_{m}^{n+1}=u_{m}^{n}-\frac{\tau}{2 h} A\left(u_{m+1}^{n}-u_{m-1}^{n}\right)-\frac{\tau^{2}}{2 h^{2}} A^{2}\left(u_{m+1}^{n}-2 u_{m}^{n}+u_{m-1}^{n}\right) .
$$

The scheme (2.11) has minimum smearing between schemes on three-point templates. However, this scheme is not monotonous and it leads to non-physical oscillations near discontinuities of the exact solution.

Hybrid scheme. Linear combination of these two schemes allows to overcome the limitations of each scheme. This combination can be written as:

$$
u_{m}^{n+1}=u_{m}^{n}-\frac{\tau}{2 h} A\left(u_{m+1}^{n}-u_{m-1}^{n}\right)+\frac{1}{2}\left((1-a) \frac{\tau}{h} \Omega^{-1}|\Lambda| \Omega+a \frac{\tau^{2}}{h^{2}} A^{2}\right)\left(u_{m+1}^{n}-2 u_{m}^{n}+u_{m-1}^{n}\right) .
$$

Here $|\Lambda|$ is a diagonal matrix of absolute eigenvalues of matrix $A$. If $a=0$ the scheme (2.12) has the first order like the scheme (2.10). If $a=1$ the scheme (2.12) has the second order like the scheme (2.11). The scheme (2.12) is hybrid one if $a$ depends on the solution local behavior. 
In this paper the local smoothness of the solution was determined from the following condition proposed by Fedorenko:

$$
\frac{\left(u_{m+1}^{n}-2 u_{m}^{n}+u_{m-1}^{n}\right)}{2} \leq K \frac{\left(u_{m+1}^{n} u_{m-1}^{n}\right)}{2} .
$$

In these calculations we assume that $K=0.5$. Parameter $a$ is 0 when inequation (2.13) is false and 1 when inequation (2.13) is true. The resulting scheme has second order for smooth solution areas and first order near discontinuities. Different advanced schemes comparison (see for instance [8] for Lax-Friedrichs scheme, Rusanov's scheme, Godunov's scheme) shows that hybrid scheme should have good balance between scheme accuracy, implementation simplicity and calculation speed. However, a detailed comparison is a separate interesting task that requires additional study.

Hybrid scheme approach allows to achieve minimum smearing in the smooth solution areas and to avoid simultaneously parasitic oscillations near discontinuities. Figure 1 shows the velocity profile for the one-dimensional problem concerning the propagation of a rectangular pulse obtained using these difference schemes. The pulse width is 40 grid points, by the time depicted the pulse traveled 200 grid points from its initial location. The calculation was performed for the Courant number $\sigma=0.7$.

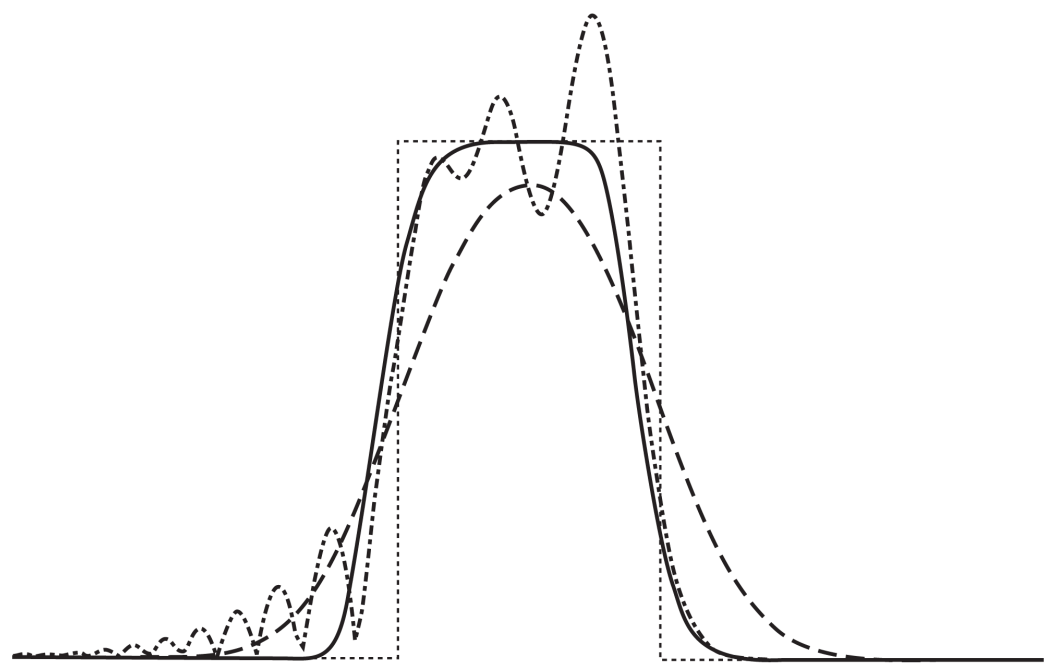

Figure 1: The problem of the rectangular pulse propagation : the dashed line corresponds to the Courant-Isaacson-Rees scheme, the dotted and dashed line corresponds to the Lax-Wendroff scheme, the solid line corresponds to the hybrid scheme and the dotted line shows the exact solution.

\subsection{Contact boundaries}

Modelling of bodies with substantially different rheological and mechanical properties requires accurate solving of the contact boundaries problem. It is important to study wave interactions 
and reflection from the boundaries. Grid-characteristic numerical methods allow to set contact boundaries conditions explicitly. This approach gives higher precision if compared with passthrough calculation.

The conditions on a contact boundary are set in the form of relations between variables at two adjacent points on the contacting surfaces (see [6]). In this paper full-adhesive

$$
v=v^{\prime}, \sigma_{n}=\sigma_{n}^{\prime}, \sigma_{\tau}=\sigma_{\tau}^{\prime}
$$

and free-sliding

$$
v_{n}=v_{n}^{\prime}, \sigma_{n}=\sigma_{n}^{\prime}, \sigma_{\tau}=\sigma_{\tau}^{\prime}=0
$$

conditions are used.

Here, variables with and without primes correspond to the opposite contacting surfaces. The indices $n$ and $\tau$ denote the normal and tangent directions, respectively.

\section{Solving different dynamic biomechanical problems}

\subsection{Modelling brain injury consequences}

The main goal of brain injury modelling in this research was to study the formation of the damage areas at early stages of cerebral trauma. It is well known in neurosurgery that damaged areas often do not coincide with the areas adjacent to the impact site. In particular, in case of the skull nape area hitting the damage is often localized in the forehead. This phenomenon referred to as a "countrecoup" can be accounted by means of numerical simulation of skull and brain wave processes.

The scheme of cerebral system that was chosen for this study is represented in Fig. 2. Quadrangular and triangular computational grids used are plotted in Fig. 3.

This research uses the model of cerebral system containing three-layer skull (two outer layers of compact bone tissue and internal layer of spongy bone tissue), a layer of liquor between skull and brain tissue, ventricles filled with liquor, membranes and gray substance.

The boundary between different layers of skull was fully adhesive. Two types of boundary conditions, free slip and adhesion, were tested for other contacts (i.e. surface between skull and gray substance). The free slip condition suits better to real biomechanical process.

Rheological parameters are summarized in the table below.

\begin{tabular}{|l|c|c|c|}
\hline Tissue & $\rho, \mathrm{kg} / \mathrm{m}^{3}$ & $\lambda, \mathrm{MPa}$ & $\mu, \mathrm{MPa}$ \\
\hline Compact bone tissue & 1600 & 7900 & 5270 \\
Spongy bone tissue & 1500 & 3975 & 2650 \\
Liquor & 1000 & 1700 & 0.001 \\
Grey substance & 1020 & 1.7 & 0.23 \\
\hline
\end{tabular}

Areas of positive and negative stresses after a strike from the left obtained as a result of modelling are presented in Fig. 4 a, b. 


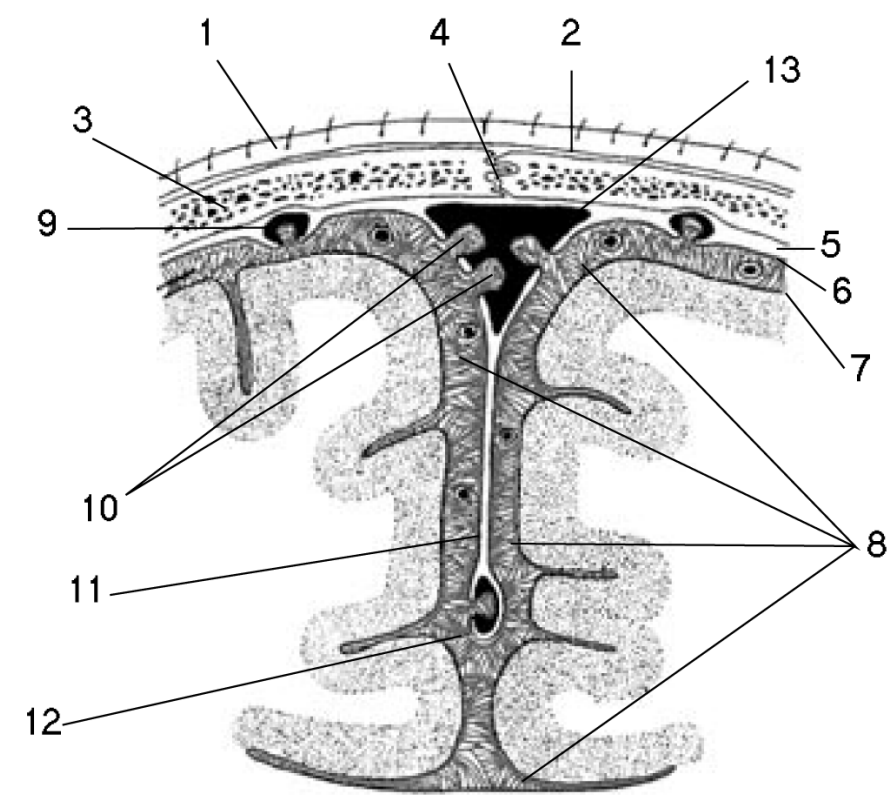

Figure 2: The scheme of cerebral system: (1) skin, (2) periosteum, (3) skull bone, (4) longitudinal seam, (5) dura mater, (6) arachnoid, (7) pia mater, (8) subarachnoidal cavity, (9) venous lacunae, (10) arachnoid granulations, (11) falx cerebrum, (12) the inferior sagittal sinus (the distances between the membranes are enlarged).
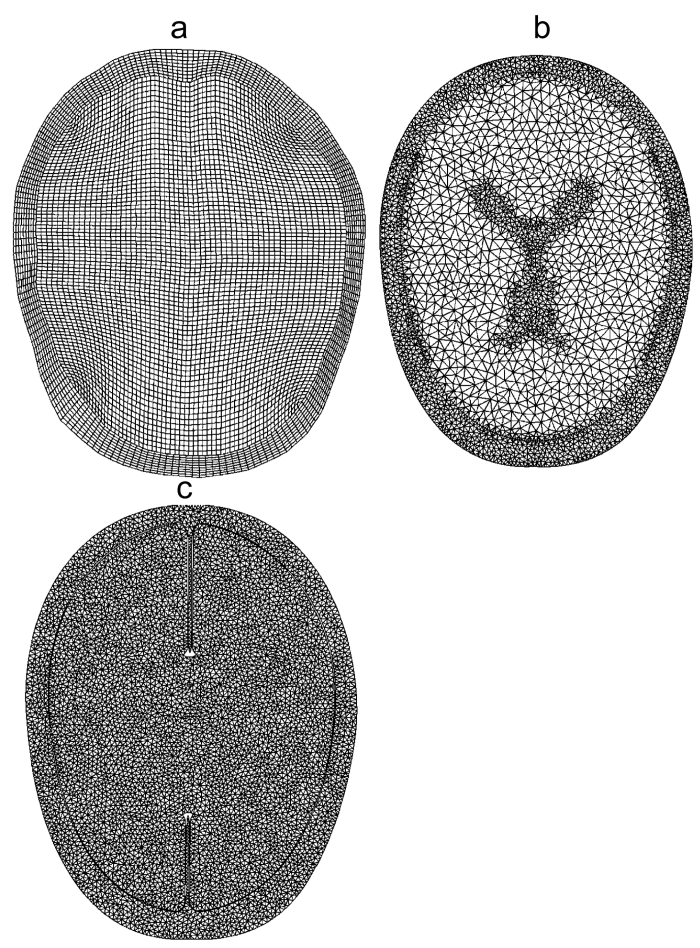

Figure 3: Quadrangular and triangular computational grids that were used in simulation. 

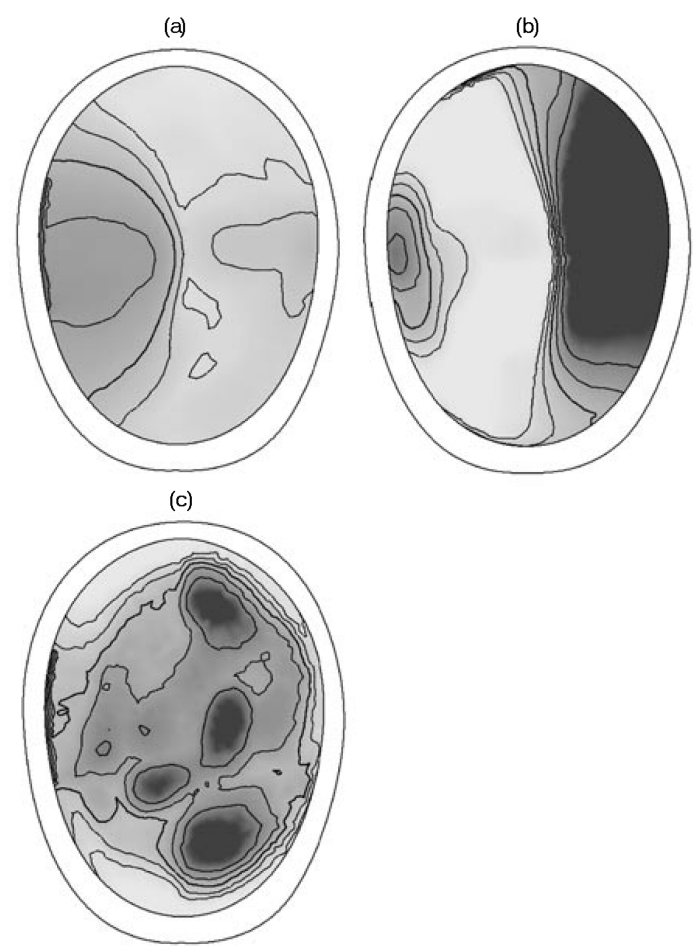

Figure 4: Areas of positive, negative and shear stresses after a strike from the left.

The hypothesis is the following: because vessels and gray substance are fibrous they should stand longitudinal strain and pressure well but transversal load can damage them much more easily. So, we can expect that the brain damage arises mostly in the areas of highest shear load.

The comparison of calculated and obtained from MRT maps brain damages is represented in Fig. 5 a, b. Areas of calculated damage refer to the zones of highest shear load. MRT map was provided by Burdenko Principal Military Clinical Hospital.

\subsection{Modelling of laser cataract extraction}

The surgery technology supposes an intracapsular crush of opaque lens nucleus by an energetic effect (ultrasound or laser radiation) without injuring of a retina or a cornea and then aspiration of masses through a self-sealing incision of width up to $3.5 \mathrm{~mm}$. A detailed understanding of the energy propagation nature in the eye depending on different parameters allows to minimize the intraoperative traumas and patients medical rehabilitation period.

This problem of the dynamic process simulation in the eye during cataract extraction can be conventionally divided into three stages. The first stage includes the computation of impulse action on the lens. The second one supposes the calculation of the acoustic pulse in vitreous body up to the retina and its impact on the retina. This part of the process has practical interest because the retina can exfoliate as a result of the pulse impact on it. The last stage supposes opaque lens masses aspiration from the anterior chamber. This research is focused on the second stage. 
(a)

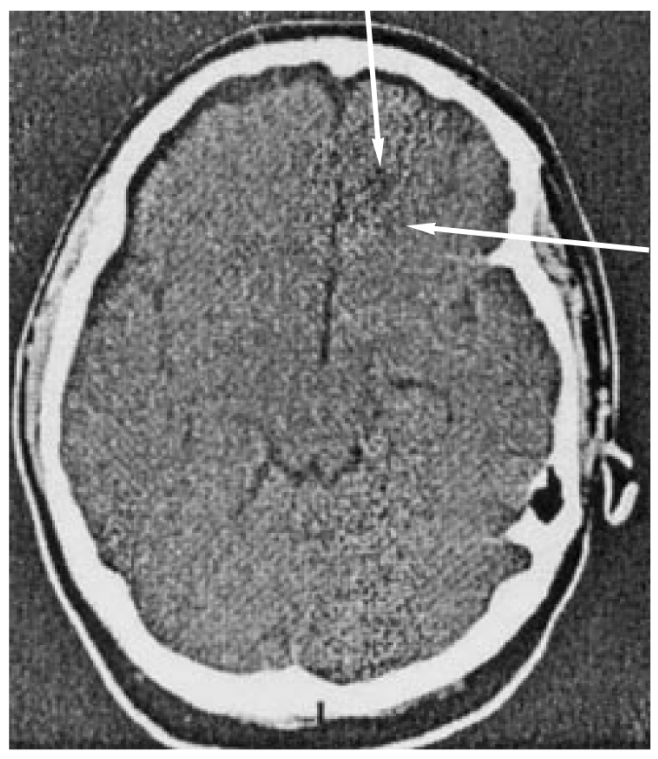

(b)

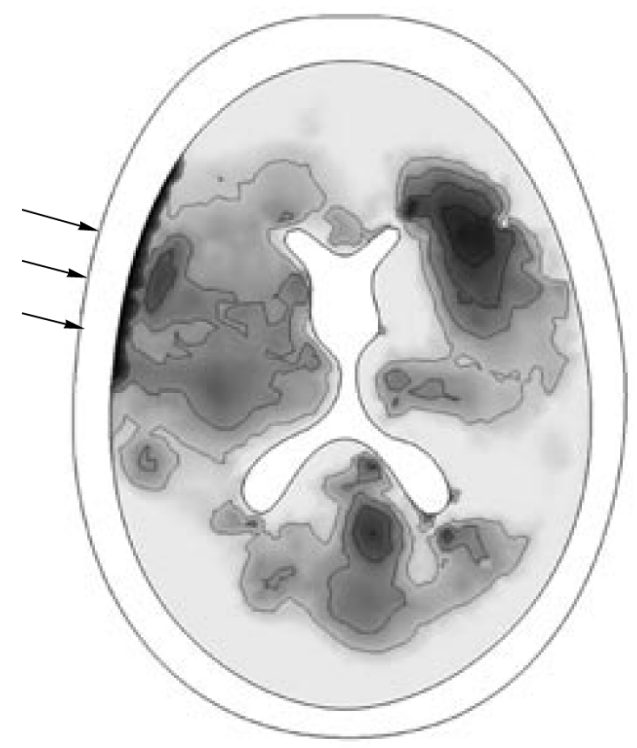

Figure 5: The comparison of computational and obtained from MRT maps brain damages.

The intensity of absorbed laser radiation is calculated according to Lambert-Beer law:

$$
\begin{aligned}
Q(r, h) & =I(r) e^{-H / h}, \\
I(r) & =I_{0} e^{-(r / R)^{2}} .
\end{aligned}
$$

Here $r$ is the radius in the cross-section of the lightguide fiber, $h$ is the depth of the laser pulse penetration, $H$ is the characteristic deepness of penetration $(H=3.2 \mathrm{~mm}), I_{0}$ is the intensity at the center of the pulse, $R$ is the lightguide fiber diameter $(R=0.3 \mathrm{~mm})$.

Since the first stage of the process was not the subject of modelling, the equivalent stress was applied for modelling pulse impact at the second stage. The laser pulse was a train with a $250 \mathrm{~ms}$ duration. The period of micropulses in the train was $12.5 \mathrm{~ms}$ and micropulse duration was $3 \mathrm{~ms}$. Only the first $250 \mathrm{~ms}$ were taken into account because perturbation intensity is noticeably attenuated after the pulse is over.

Pulse propagation through the lens and the vitreous humour to the retina was modelled as described above. Computational grid and velocity field are shown at Fig. 6.

The distribution of ocular media particle speeds is shown at Fig. 7 at time moments $t_{1}=$ $6.24 \mathrm{~ms}$, when the perturbation reached the iris and $t_{2}=23.6 \mathrm{~ms}$ when the perturbation is reflected from the posterior surface of the eye. It can be noted that the movement of ocular media has a rather complicated character. At the initial moment during the pulse an expansion of bio-media occurs, after the pulse is over, the pressure in the lens zone becomes less than in its surrounding areas. It induces changes of speed direction and a subsequent eye contraction as a consequence. So, the eye is periodically expanded and constricted during the operation. Tissue ruptures as well as cavitation processes are possible in the sites of expansion. In addition, reflected waves from ocular materials borders influence the wave picture. 

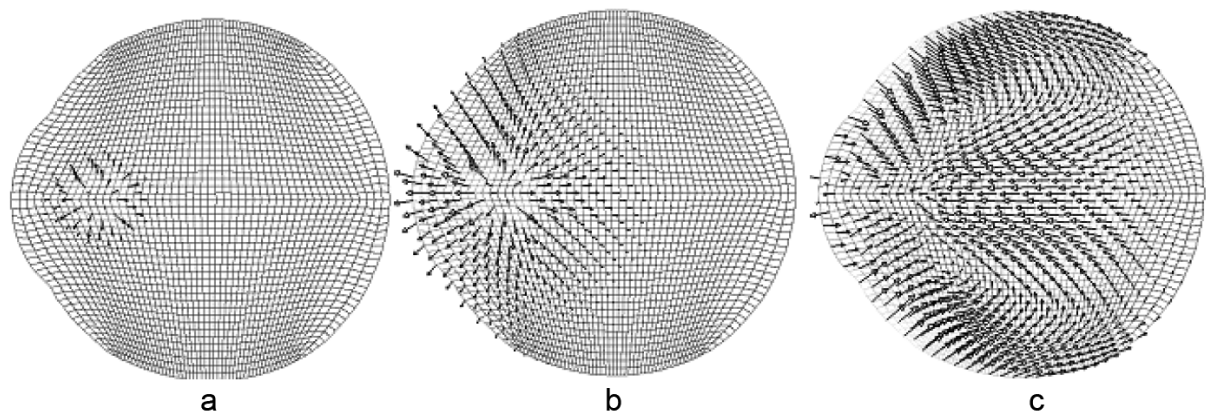

Figure 6: Computational grid and velocity field.
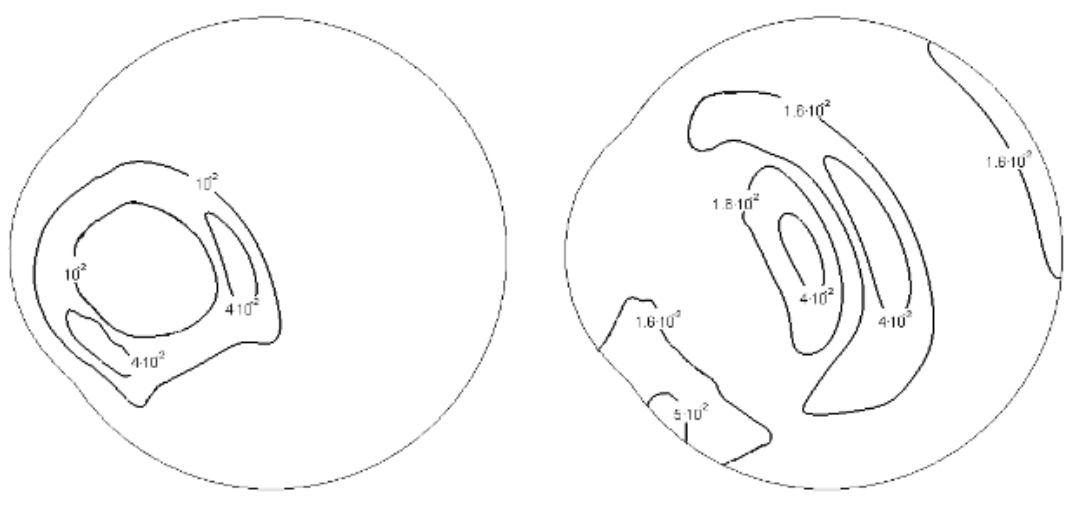

Figure 7: Isolines of speed modulus. Numerical characteristic of speed are indicated in $\mathrm{m} / \mathrm{sec}$. 
As the result the picture of potential zones of ocular structure damage risk is rather complicated (see Fig. 8).

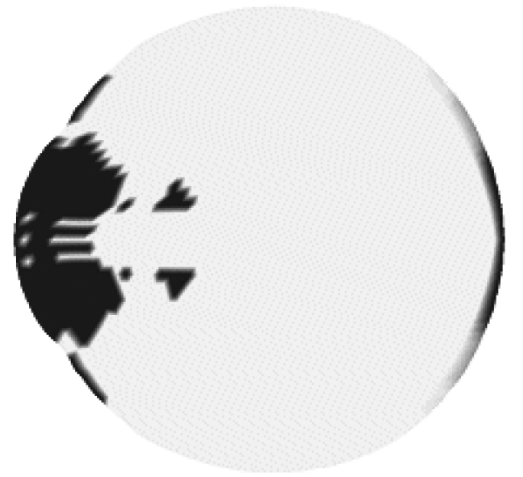

Figure 8: Zones of risk of the ocular structure damage.

An eye lens pressure was also modelled using modern FEM software (ANSYS). The results are presented at Fig. 9.

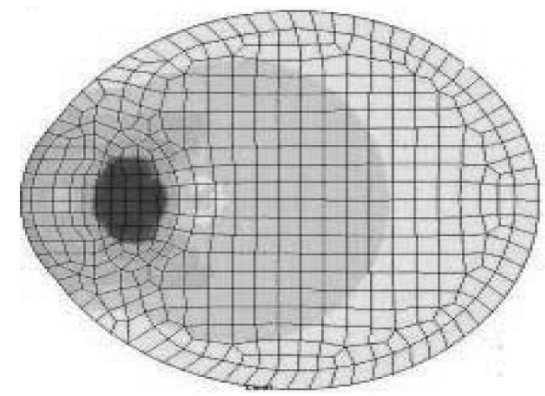

Figure 9: Calculation of pressure in eye lens using FEM and computational grid.

It is worth paying attention to the fact that finite-element method identifies the zone of the most probable damage near the lightguide fiber. Method of characteristics allows to find also a possible damage zone near the retina and a number of small additional risk zones in the eye frontal part. These additional zones are caused by wave fronts interactions during initial fast dynamic part of overall process and can not be found using static or quasi-static modelling methods.

\section{Acknowledgments}

The authors are grateful to the researchers from Sklifosovsky Scientific Research Institute of Emergency Medicine, Burdenko Principal Military Clinical Hospital and Fyodorov Eye Microsurgery Institution for cooperation, useful comments and suggestions. 


\section{References}

[1] J.H. Adams, D.I. Graham, T.A. Genmarelli. Head injury in man and experimental animals: neuropathology. Acta Neurochir., 32 (1975), 15-30.

[2] L. Zhang, K.H. Yang, A.I. King. Comparison of brain responses between frontal and lateral impacts by finite element modelling. J. Neurotrauma, 18 (2001), 21-30.

[3] A.H. Kuijpers, M.H. Claessens, A.A. Sauren. The influence of different boundary conditions on the response of the head to impact: a two-dimensional finite element study. J. Neurotrauma, 12 (1995), 715-724.

[4] V. Novatskii. Elasticity theory. Nauka, Moscow, 1975 (in Russian).

[5] K.M. Magomedov, A.S. Kholodov. Grid characteristic methods. Nauka, Moscow, 1988 (in Russian).

[6] I.B. Petrov, A.G. Tormasov, A.S. Kholodov. On the numerical study of unsteady processes in layered solids. Izv. Akad. Nauk SSSR, Ser. Mekh. Tverd. Tela, 4 (1989), 89-95.

[7] I.B. Petrov, A.S. Kholodov. Numerical study of some dynamic problems in mechanics of deformable solids by the grid characteristic method. Zh. Vych. Mat. Mat. Fiz., 24 (1984), 722-739.

[8] J.A. Trangenstein. Numerical solution of hyperbolic partial differential equations. Cambridge University Press, New York, 2007. 